\title{
Whole Brain Radiotherapy Combined with Stereotactic Radiosurgery versus Stereotactic Radiosurgery Alone for Brain Metastases
}

\author{
Yasemin Guzle Adas*, Omer Yazici, Esra Kekilli, Ebru Atasever Akkas, Ebru \\ Karakaya, Ali Riza Ucer, Gulcin Ertas, Tamer Calikoglu, Yesim Elgin, Gonca \\ Altinisik Inan, Ali Mert Kocer, Yildiz Guney
}

\begin{abstract}
Background: The aim of this study was to evaluate the effect of whole brain radiotherapy (WBRT) combined with streotactic radiosurgery versus stereotactic radiosurgery (SRS) alone for patients with brain metastases. Materials and Methods: This was a retrospective study that evaluated the results of 46 patients treated for brain metastases at Dr.Abdurrahman Yurtaslan Ankara Oncology Training and Research Hospital, Radiation Oncology Department, between January 2012 and January 2015. Twenty-four patients were treated with WBRT+SRS while 22 patients were treated with only SRS. Results: Time to local recurrence was 9.7 months in the WBRT+SRS arm and 8.3 months in SRS arm, the difference not being statistically significant $(p=0.7)$. Local recurrence rate was higher in the SRS alone arm but again without significance $(p=0,06)$. Conclusions: In selected patient group with limited number (one to four) of brain metastases SRS alone can be considered as a treatment option and WBRT may be omitted in the initial treatment.
\end{abstract}

Keywords: Brain metastases - whole brain radiotherapy - stereotactic radiosurgery

Asian Pac J Cancer Prev, 16 (17), 7595-7597

\section{Introduction}

Metastatic brain tumors are the most common types of brain tumors and ten times more than primary brain tumors (Akhavan et al., 2014). Upward trend of brain metastases may be related with prolonged overall survival of cancer patients or the development in the imaging modalities (Song et al., 2013). The prognosis of patients with brain metastases are generally poor even be about 4 weeks without treatment so treatment of brain metastases can not be ignored by the physicians (Sundstrom et al., 1998).

There are various treatment modalities for brain metastases, including surgery, radiotherapy and systemic therapy. Radiotherapy is the mainstay of the treatment WBRT being the most common technique (Phungrassami et al., 2015).

In 1970s, the first option for radiotherapy of brain metastases was WBRT but in recent years the use of WBRT decreased mainly because of the late neurological toxicities such as memory loss, emotional dysfunction, dementia, stupor and coma (Duan et al., 2014; Abe et al., 2012; Mctyre et al., 2013). Because of these adverse effects SRS has been popular. SRS has some advantages. First it is not an invasive procedure like surgery; second, compared with WBRT, less healty tissue can be exposed
(Linskey et al., 2010). But a higher rate of local tumor reccurence was shown by SRS monotherapy (Aoyama et al., 2006).

The efficiency of WBTR plus SRS and SRS alone still remains unclear. In this retrospective trial we evaluated the outcomes of WBRT+SRS and SRS alone in metastatic brain tumors.

\section{Materials and Methods}

This is a retrospective study that evaluated the results of 46 patients treated for brain metastases at Dr. Abdurrahman Yurtaslan Ankara Oncology Training And Research Hospital Radiation Oncology Department between January 2012 and January 2015. Patients had limited number of (one to four) metastatic brain lesions. Twenty-four patients were treated with WBRT+SRS while twenty-two patients were treated with only SRS. Twenty-six patients had only brain metastases. Fifteen of these patients were in only SRS group an eleven in SRS+WBRT group. Nine patients had limited extracranial disease and the others had diffuse extracranial disease. In WBRT group WBRT dose was 30 Gy (300 cGy x 10 fractions). In WBRT+SRS group WBRT dose was 25 Gy (250cGy x 10 fractions) in 3 patients and 30 Gy 
Table 1. Patient Characteristics

\begin{tabular}{llc}
\hline Age & median 54 & $\begin{array}{c}\text { me } \\
\text { (range 31-73) }\end{array}$ \\
\hline Sex & Female & 16 \\
& Male & 30 \\
Primary Diagnosis & Lung & 28 \\
& Breast & 13 \\
Extra-cranial metastases & Others & 5 \\
& Yes & 20 \\
Number of brain metastases & No & 26 \\
& 1 lesion & 19 \\
& 2 lesion & 13 \\
\multirow{5}{*}{ Radiotherapy } & 3 lesion & 5 \\
& 4 lesion & 9 \\
& WBRT & 22 \\
& WBRT+SRS & 24 \\
\hline
\end{tabular}

(300cGyx10fractions) in 21 patients. After WBRT, boost was applied with SRS. The boost dose was 15-24 Gy in 1-3 fractions. In SRS alone group, dose was 18-24 Gy in one to three fractions. Patients were evaluted with cranial MRI every two months after the completion of radiotherapy. If the patient had neurological symptoms cranial MRI was performed earlier. The groups were compared in terms of local recurrence rates and time to local reccurence.

\section{Statistical method}

Data analysis was performed using SPSS 16 for Windows. Time to local recurrence was assesed using Kaplan-Meier log rank test in univarian analysis and MANOVA test in multivarian analysis. Local recurrence rate of both groups were also assesed by using chi-square test. Results were admitted statistically significant for $\mathrm{p}<00.05$.

\section{Results}

Median age was 54 (range 31-73). Primary diagnosis were lung cancer (28 patients), breast cancer (13 patients), and others (5 patients). The patient charecteristics were summarized in table 1 . Time to local reccurence was 9 , 7 months in WBRT+SRS arm and 8, 3 months in SRS $\operatorname{arm}(\mathrm{p}=0,7)$ and was not statistically important. Local recurrence was observed on 10 of 24 patients (41.7\%) in WBRT+SRS arm and 15 of 22 patients $(68.2 \%)$ in SRS arm. Local recurrence was higher in SRS alone arm but was not statistically important $(p=0,06)$. The effect of age (older than 50 years vs. younger than 50 years), sex, number of metastatic brain lesions (one lesion vs more than one lesion), status of primary disease (stable primary vs unstable primary) were also considered. There was no effect of these factors on local reccurence .

\section{Discussion}

Metastatic brain tumors aren't the malignancy originating from the nerve tissue, but its morbidity is the highest among malignant brain tumors and would be more complicated in treatments compared with the primary tumors in brain (De Angelis et al., 2001). Treatment method of brain metastases can vary according to the patient's performance status, the extent of primary disease and the number of metastatic lesions in brain (Fabi et al., 2011).

In a randomized trial (Patchell et al., 1990) adjuvant WBRT after surgical resection of single brain metastases was assessed. This study randomized 95 patients with a single brain metastases to receive surgery plus adjuvant WBRT or surgery alone. Patients were observed with MRI. They reported that patients with single brain metastases who receive surgical resection plus radiotherapy live longer; have fewer recurrences in brain than similar patients treated with radiotherapy alone. In a systemic review (Gaspar et al., 2010) surgical resection followed by WBRT, as compared to WBRT alone, was offered as standart treatment modality in patients with good performance status, single brain metastases and limited extracranial disease.

In EORTC 22952-26001 trial (Kocher et al., 2010) the role of adjuvant WBRT after surgery or radiosurgery was assessed. Threehundred-fiftynine patients with one to three brain metastases of solid tumors treated with surgery or radiosurgery and randomly assigned to adjuvant WBRT or observation. Overall survival was similar in the WBRT and observation arms (median, 10.9 vs. 10.7 months). WBRT reduced the 2-year relapse rate both at initial sites (surgery: $59 \%$ to $27 \%, \mathrm{p}<0.001$; radiosurgery: $48 \%$ to $33 \%, \mathrm{p}=0.023)$.

In a phase III Japan trial (Aoyama et al., 2006) that randomly assigned 132 patients with one to four metastases to radiosurgery alone or to radiosurgery plus WBRT; they reported that adjuvant WBRT after radiosurgery or surgery reduces the intracranial relapses. The 12-month brain tumor recurrence rate was $46.8 \%$ in the WBRT + SRS group and $76.4 \%$ for SRS alone group $(\mathrm{p}<0.001)$. In this study we did not find any difference between WBRT+SRS and only SRS group in terms of intracranial relapses.

In recent studies (Aoyama et al., 2006; Kocher et al., 2010) intracranial relapse was reduced by adding WBRT to SRS, but no overall survival advantage was reported by adding WBRT to SRS. In this study we did not observe statistical difference on intracranial recurrence rate or time to local reccurence between two groups. We observed more local recurrences in SRS alone arm but that was not statistically important. Both EORTC 22952-26001 and Japan trials are randomized prospective trials with large number of patients. In this retrospective trial we had a small number of patients.

In conclusion, our findings demonstrated that SRS alone without WBRT did not increase brain tumor recurrence and in selected patient group with limited number of brain metastases (one to four metastases) SRS alone can be considered as a treatment option and WBRT may not be included in the initial treatment. But this is a retrospective study with limited number of patients and more studies are needed to research this issue.

\section{References}

Abe E, Aoyama H (2012). The role of whole brain radiation therapy for management of brain metastases in era of 
stereotactic radiosurgery. Curr Oncol Rep, 14, 79-84

Akhavan A, Binesh F, Heidari S (2014). Survival of metastatic patients in Yazd, Iran. Asian Pac J Cancer Prev, 15, 3571-4

Aoyama H, Tago M, Kato N, et al (2006). Neurocognitive function of patients with brain metastasis who received either whole brain radiotherapy plus stereotactic radiosurgery or radiosurgery alone. Int J Radiat Oncol Biol Phys, $\mathbf{6 8}$, 1388-95

Duan L, Zeng R, Yang KH, et al (2014) Whole Brain Radiotherapy Combined with Stereotactic Radiotherapy Versus Stereotactic Radiotherapy Alone for Brain Metastases: a Meta-analysis. Asian Pac J Cancer Prev, 15, 911-5

Fabi A, Felici A, Metro G, et al (2011). Brain metastases from solid tumors: disease outcome according to type of treatment and therapeutic resources of the treating center. J Exp Clin Cancer Res, 30, 10.

Gaspar EL, Mehta MP, PAtchell RA, et al (2010). The role of whole brain radiation therapy in the management of newly diagnosed brain metastases: a systematic review and evidence-based clinical practice guideline. J Neurooncol, 96, 17-32

Kocher M, Soffietti R, Abacioglu U, et al (2011). Adjuvan Whole-Brain radiotherapy versus observation after radiosurgery or surgical resection of one to three cerebral metastases: results of the EORTC 22952-26001 Study. $J$ Clin Oncol, 29, 134-141

Linskey ME, Andrews DW, Asher AL, et al (2010). The role of stereotactic radiosurgery in the management of patients with newlly diagnosed brain metastases: a systemic review and evidence-based clinical practice guidline. J Neurooncol, 96, 45-68

Mctyre E, Scott J, Chinnaiyan P(2013). Whole brain radiotherapy for brain metastasis. Surg Neurol Int, 4, 236-44

Patchell RA, Tibbs PA, Walsh JW et al (1990). A randomized trial of surgery in the treatment of single metastases to the brain. $N$ Engl J med, 322, 494-500

Phungrassami T, Sriplung H (2015) Radiotherapy for brain metastases in southern Thailand: workload, treatment pattern and survival. Asian Pac J Cancer Prev, 16, 1435-42.

Song WG, Wang YF, Wang Rl, et al (2013). Therapeutic Regimens and Prognostic Factors of Brain Metastatic Cancers. Asian Pac J Cancer Prev, 14, 923-7

Sundström JT, Minn H, Lertola KK et al.(1998) Prognosis of patients treated for intracranial metastases with whole-brain irradiation, Ann Med, 30, 296-9. 